\title{
Effective hepatic artery chemoembolization for advanced hepatocellular carcinoma with multiple tumor thrombi and pulmonary metastases: A case report
}

\author{
DE-JIA HUANG ${ }^{1,2}$, YAN-HAO LI ${ }^{1}$, YAO-CHANG LUO ${ }^{2}$, JUN-ZHEN HUANG ${ }^{2}$ and HAI-YUAN HE ${ }^{2}$ \\ ${ }^{1}$ Department of Interventional Radiology, Nanfang Hospital, Southern Medical University, Guangzhou, Guangdong 510515; \\ ${ }^{2}$ Department of Interventional Radiology, The First Affiliated Hospital of Guangxi Traditional Chinese Medical University, \\ Nanning, Guangxi 530021, P.R. China
}

Received February 28, 2015; Accepted January 5, 2016

DOI: $10.3892 / \mathrm{ol} .2016 .4846$

\begin{abstract}
Advanced hepatocellular carcinoma (HCC) with tumor thrombi invading the portal vein and extending into the right atrium (RA) through the hepatic vein is regarded as a terminal-stage condition. Intracardiac tumor thrombus and treatment via liver resection has been reported in the current literature, but results from this therapeutic approach remain unsatisfactory. The present study describes a rare case of HCC with metastatic portal vein, middle hepatic vein, inferior vena cava (IVC) and RA tumor thrombi, and pulmonary metastases. A 29-year-old woman was admitted to The First Affiliated Hospital of Guangxi Traditional Chinese Medical University (Nanning, China) subsequent to experiencing right upper quadrant abdominal pain. Following diagnosis, based on computed tomography analysis and laboratory data, the patient underwent an initial transcatheter arterial chemoembolization (TACE) treatment using fluorouracil (5-FU), pirarubicin, mitomycin $\mathrm{C}$, Lipiodol and sodium alginate microball (KMG). At 1 month post-treatment, serum $\alpha$-fetoprotein levels remained at $>1,000 \mathrm{ng} / \mathrm{ml}$. Subsequently, the patient underwent a second TACE treatment. At 1 month after the second treatment, the abdominal pain had been alleviated and the serum $\alpha$-fetoprotein levels were reduced to $<20 \mathrm{ng} / \mathrm{ml}$. Imaging analysis indicated a marked reduction in tumor burden in the liver and the hepatic vein and IVC tumor thrombi. Furthermore, the portal vein and RA tumor thrombi, and the pulmonary metastases had disappeared. At 40 months after the second TACE therapy, the patient remains alive without any signs of recurrence. The present case demonstrates that the administration of TACE,
\end{abstract}

Correspondence to: Dr Yan-Hao Li, Department of Interventional Radiology, Nanfang Hospital, Southern Medical University, 1838 Guangzhou Avenue North, Guangzhou, Guangdong 510515, P.R. China

E-mail: liyanhao@fimmu.com

Key words: hepatocellular carcinoma, tumor thrombus, right atrium, pulmonary metastases, hepatic artery chemoembolization, pirarubicin, sodium alginate microball using 5-FU, pirarubicin, mitomycin $\mathrm{C}$, Lipiodol and $\mathrm{KMG}$, functions as an effective treatment in cases of unresectable advanced HCC presenting with pulmonary metastases and extensive tumor thrombi in the IVC, the RA and one branch of the portal vein.

\section{Introduction}

Hepatocellular carcinoma (HCC) is the third highest cause of cancer-associated mortality worldwide following stomach and lung cancer (1). Liver cancer is the sixth most common cancer worldwide, accounting for $5.7 \%$ of all novel cancer cases. A total of $\sim 82 \%$ of liver cancer cases occur in developing countries, with $55 \%$ occurring in China alone. There is a high incidence rate of HCC in Asia, and particularly so in China due to endemic hepatitis B and C $(2,3)$. However, only $<10 \%$ of patients in Asia and $\sim 30 \%$ of patients in the West are eligible at diagnosis for potentially curative treatments, including resection or liver transplantation $(4,5)$ and radiofrequency ablation $(6,7)$.

Despite regular medical examinations, certain patients that are diagnosed with HCC also present with extensive tumor thrombi, typically extending into the inferior vena cava (IVC) and right atrium (RA) through the hepatic vein $(8,9)$; this condition is considered as terminal-stage HCC. Intracardiac tumor thrombus and liver resection treatment have been reported in the current literature, alongside alternative therapeutic approaches (i.e., radiotherapy), but long-term results from such treatments remain unsatisfactory (10-15). Transcatheter arterial chemoembolization (TACE) has since been recommended for the treatment of these patients (16).

The present study describes the case of a patient with HCC and metastatic tumor thrombi of the right branch of the portal vein, inferior hepatic vein, IVC and RA, with pulmonary metastases. Following two effective TACE treatments, the patient remains alive at 40 months follow-up, without any signs of recurrence.

\section{Case report}

A 29-year-old woman with hepatitis B was referred to the First Affiliated Hospital of Guangxi Traditional Chinese Medical 

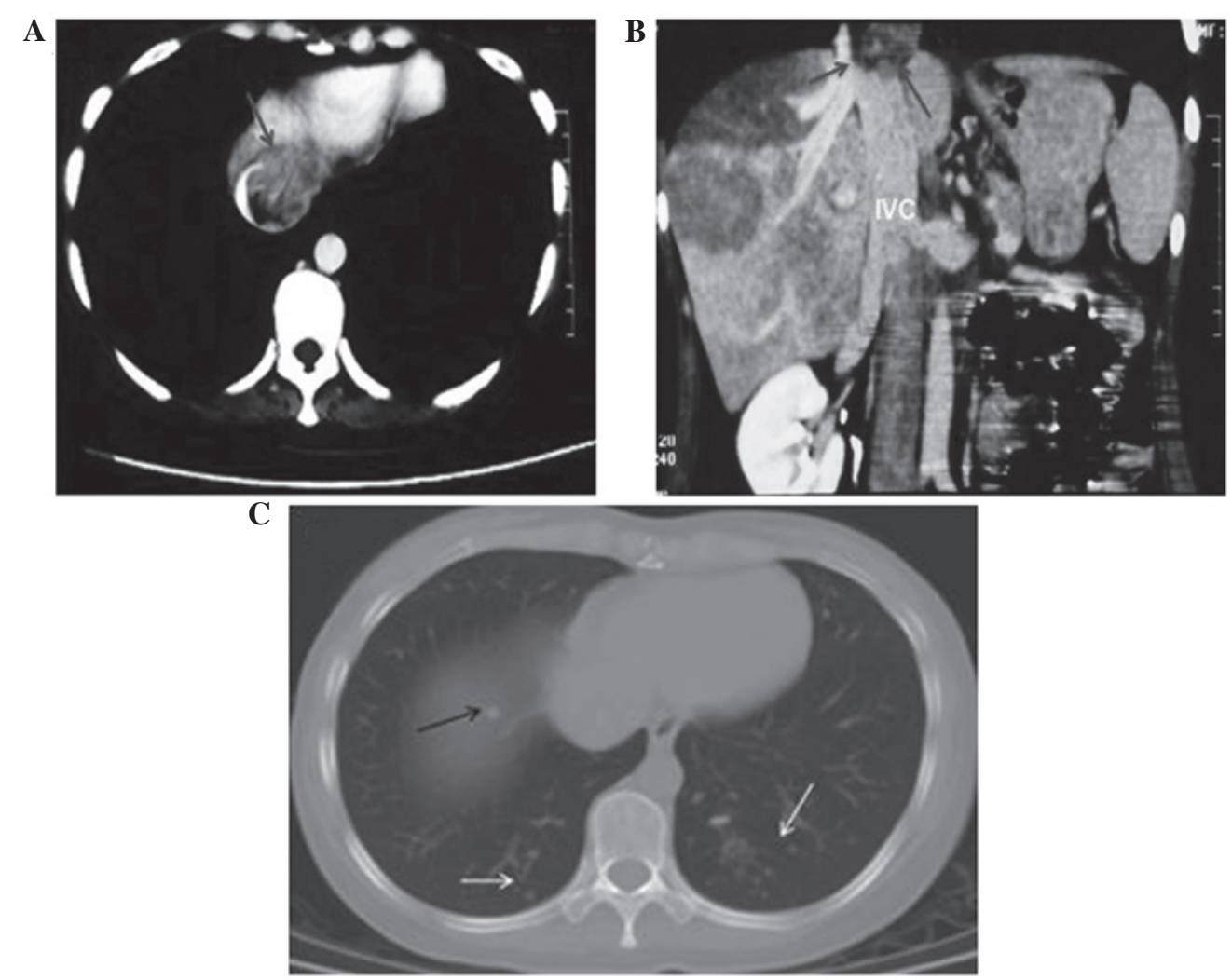

Figure 1. Abdominal CT prior to transcatheter arterial chemoembolization. Arterial phase of the CT scan, (A) cross-sectional scan and (B) coronal scan, presenting a well-defined, lobulated filling defect in the right atrium (arrows) and an irregular enhancement. (C) Chest CT scan exhibiting several solitary nodules (indicated by the black and white arrows) in the bilateral lower lobe lung, all of which were $<0.5 \mathrm{~cm}$ in diameter. CT, computed tomography; IVC, inferior vena cava.

University (Nanning, China) on September 5, 2011, presenting with a 1-month history of right upper quadrant abdominal pain and abnormal shadows in the liver on an abdominal computed tomography (CT) scan (performed using a BrightSpeed Elite 16 slice CT scanner; GE Healthcare Life Sciences, Chalfont, UK) that had been performed prior to referral. Physical examination identified mild abdominal tenderness in the upper right quadrant, with no rebound tenderness. Cardiovascular, respiratory and neurological examinations were normal. The family history of the patient was non-contributory. Laboratory analysis provided the following results: Platelets, $201 \times 10^{9} / 1$ (normal range, $100-300 \times 10^{9} / 1$ ); serum aspartate aminotransferase, $107 \mathrm{U} / 1$ (normal range, $<40 \mathrm{U} / 1$ ); alanine aminotransferase, $94 \mathrm{U} / 1$ (normal range, $<40 \mathrm{U} / \mathrm{l}$ ); total bilirubin, $9.1 \mu \mathrm{mol} / 1$ (normal range, $<20 \mu \mathrm{mol} / \mathrm{l}$ ); albumin, $31.7 \mathrm{~g} / 1$ (normal range, 34-54 g/l); and prothrombin time, $14.5 \mathrm{sec}$ (normal range, 12-14 sec). Screening for hepatitis B was positive for all antigens and negative for antibodies. The serum level of $\alpha$-fetoprotein (AFP) was $>1,000 \mathrm{ng} / \mathrm{ml}$ (normal range, $<20 \mathrm{ng} / \mathrm{ml}$ ). No abnormalities were observed on the electrocardiogram.

An abdominal enhanced CT scan was performed at the Guangxi Tumor Hospital (Nanning, China) hospital 4 days prior to admission, and revealed multifocal liver lesions of various sizes, ranging from $2.0-8.5 \mathrm{~cm}$ in diameter in segments 4, 5, 7 and 8 , and a right portal vein tumor thrombus extending through the middle hepatic vein and IVC into the RA (Fig. 1A and B). A CT scan of the chest identified several solitary nodules in the bilateral lower lobes of the lungs, all of which were $<0.5 \mathrm{~cm}$ in diameter (Fig. 1C). Echocardiography was not performed at that time, as the tumor thrombus could not be clearly observed on the CT scan.

A selective hepatic angiogram was performed during the first TACE treatment and revealed three large hypervascular areas in the right lobe of the liver supplied by the right hepatic artery (Fig. 2). These hypervascular areas were typical of HCC, and extended into the IVC and RA through the middle hepatic vein. The feeding arteries of the tumor thrombus and the tumor itself originated from the left and right hepatic arteries. Therefore, following imaging analysis, the patient was diagnosed with HCC complicated by portal vein, middle hepatic vein and metastatic right atrial tumor thrombi, with pulmonary metastases (no biopsy was performed to confirm the exact nature of these masses).

In September 2011, the patient underwent the first 6-week cycle of TACE treatment with fluorouracil (5-FU; $500 \mathrm{mg}$ ), pirarubicin (20 mg), mitomycin C (10 mg), super-liquid iodized oil (Lipiodol ${ }^{\circledR}$; $9.5 \mathrm{ml}$; Guerbet, Aulnay-sous-Bois, France) and sodium alginate microball (KMG; $1.0 \mathrm{~g}$; Beijing Shengyiyao Science \& Technology Development Co., Ltd., Beijing, China). At 1 month after the initial TACE treatment, serum AFP levels remained at $>1,000 \mathrm{ng} / \mathrm{ml}$. No complications of TACE were observed, and the symptoms were partially relieved. CT revealed necrosis of a few of the tumor masses, but a number of active lesions remained. Therefore, in October 2011, the patient underwent a second 6-week cycle of TACE treatment with 5-FU (500 mg), pirarubicin $(10 \mathrm{mg})$, mitomycin C (10 mg), super-liquid iodized oil $(2 \mathrm{ml})$ and KMG $(0.3 \mathrm{~g})$. 

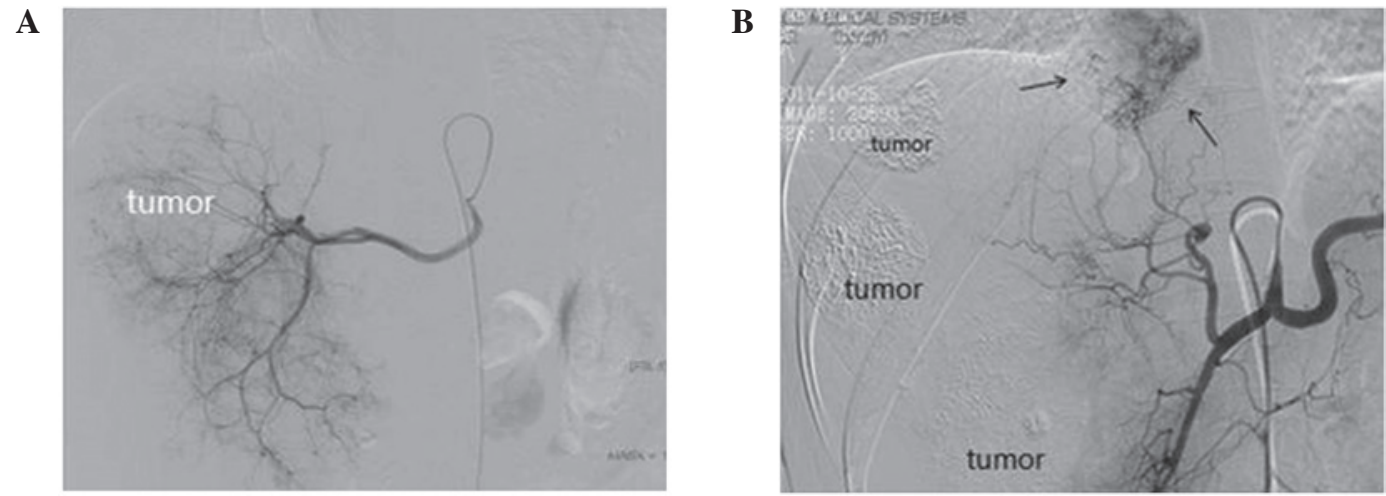

Figure 2. Angiographic image of the patient. (A) During the first TACE treatment, three large hypervascular areas of tumor staining were observed at the right liver lobe supplied by the right hepatic artery, which were typical of hepatocellular carcinoma. (B) The second TACE treatment revealed that the Lipiodol remained in the tumor of the right lobe liver. The tumor thrombus of the RA was a hypervascular lesion supplied by the right hepatic artery. The artery entered the RA by passing the inferior vena cava, and was of a 'grating'-like type (arrow). TACE, transcatheter arterial chemoembolization; RA, right atrium.

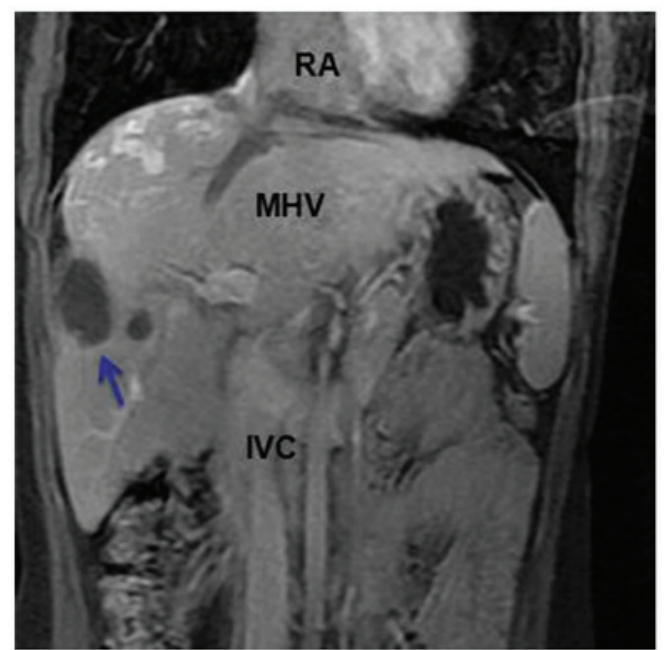

Figure 3. Magnetic resonance imaging demonstrating shrinkage of the right atrium tumor thrombus prior to its eventual disappearance. The liver tumors (arrow) and the tumor thrombus in the inferior vena cava and middle hepatic vein are also markedly reduced in size. RA, right atrium; $\mathrm{MHV}$, middle hepatic vein; IVC, inferior vena cava.

At 1 month after the second TACE treatment, the right upper quadrant abdominal pain had disappeared. The serum AFP levels gradually decreased and fell within the normal range $(<20 \mathrm{ng} / \mathrm{ml})$. At a total of 4 months after the second TACE treatment, CT revealed that the lung metastases had disappeared. A magnetic resonance imaging (MRI) scan was performed (using a Signa HDxt 1.5T MRI scanner; GE Healthcare Life Sciences) 14 months after the second TACE treatment in order to detect any lesions that had been missed on the CT scan. MRI indicated that there was no active lesion, that the tumor thrombus in the right branch of the portal vein and RA had disappeared, and that the liver tumors and the tumor thrombus in the IVC and middle hepatic vein had markedly decreased in size (Fig. 3). Subsequent to therapy, the patient was not administered any further drug treatments, as the liver function remained normal. The patient has been regularly followed up, and at 40 months post-treatment is currently alive and well, exhibiting no signs of tumor progression and with excellent quality of life. The present study was approved by the Ethics Committee of the First Affiliated
Hospital of Guangxi Traditional Chinese Medical University. Full informed consent was obtained from the patient.

\section{Discussion}

$\mathrm{HCC}$ is particularly prevalent in East Asia due to the high incidence of hepatitis B and C infection $(2,3,17)$. Liver transplantation or hepatic resection is limited to a small number of HCC patients (18). Advanced HCC presenting with extensive intravascular tumor thrombi is a rare condition and is considered as a terminal stage of HCC (19-21). The majority of patients with terminal-stage $\mathrm{HCC}$ receive no anticancer treatment and typically succumb to the disease within a few months of diagnosis, with a median survival time of 2.0-3.5 months $(11,22)$. The most appropriate treatment for patients with HCC metastases to the RA remains controversial $(8-10,12,13,15,16,19,23)$. As the majority of patients are already in the advanced stage of the disease at diagnosis, aggressive treatment is not recommended. It was previously reported that the survival time following surgical removal of intra-atrial HCC ranged from 18 days to 56 months (median, 11 months), depending on the characteristics of each individual patient (23). However, regarding patients with HCC and tumor thrombi of the hepatic vein, portal vein and RA, plus pulmonary metastases, no effective therapy has yet been reported, with surgical resection being unsuitable for such patients. Alternative non-surgical therapies, including percutaneous ethanol injection, microwave coagulation therapy and irradiation, have also produced unsatisfactory results in patients with advanced HCC $(5,11,21,24,25)$. Therefore, no standard regimens have yet been approved.

TACE has become the most successful treatment for advanced HCC, providing a survival benefit for selected patients (26). The treatment is extensively used in patients with HCC invading the portal vein, and also in those who are not suitable for surgery (27). Hepatic artery infusion chemotherapy is particularly effective in treating the disease and may offer survival benefits, even when the patient presents with extrahepatic metastases (28).

In the present study, TACE with 5-FU, pirarubicin, mitomycin C, Lipiodol and KMG achieved a marked anticancer effect, not only on the primary tumor, but also on the tumor 
thrombi in the portal and hepatic veins and the RA, and on the pulmonary metastases. The patient is currently alive at 40 months post-treatment, which is far longer than the 2.0 to 3.5-month survival time typically observed in patients with this disease; however, it should be noted that the survival time of the patient is within the 18-day to 56-month range reported with the use of other treatment strategies. Emulsions of Lipiodol and chemotherapy have been reported to remain in the hepatic tissues for a long period of time after infusion through the portal vein and hepatic artery (29). The use of KMG induces permanent hepatic artery embolization and leads to long-term anticancer drug retention in the tumor.

A previous systematic review indicated that TACE improves the survival time of patients with unresectable HCC, and that it should be considered as a standard treatment (4). Another previous study in patients with HCC and thrombi of the IVC and RA treated with TACE reported that the median survival time in responders was 13.5 months (range, 1.5-79.7 months) compared with 3.3 months (range, 2.1-24.3 months) in non-responders (16). However, the tumor burden in these patients was less than that observed in the present case. Further trials should be conducted to assess the efficacy of TACE in such patients, however, the rarity of the disease would be a limiting factor on the number of trials that may be undertaken. Nevertheless, the few reported cases, including the present case, suggest that TACE may be an appropriate and successful approach to treat HCC patients presenting with multiple venous thrombi and distant metastases.

In conclusion, the current case may suggest that TACE, using 5-FU, pirarubicin, mitomycin C, Lipiodol and $\mathrm{KMG}$, may serve as an effective treatment in patients presenting with unresectable advanced HCC with pulmonary metastases, and with extensive tumor thrombi in the IVC, the RA and one branch of the portal vein.

\section{References}

1. Siegel R, Ma J, Zou Z and Jemal A: Cancer statistics, 2014. CA Cancer J Clin 64: 9-29, 2014.

2. Llovet JM, Fuster J and Bruix J: Prognosis of hepatocellular carcinoma. Hepatogastroenterology 49: 7-11, 2002.

3. Tan CK, Law NM, Ng HS and Machin D: Simple clinical prognostic model for hepatocellular carcinoma in developing countries and its validation. J Clin Oncol 21: 2294-2298, 2003.

4. Llovet JM and Bruix J: Systematic review of randomized trials for unresectable hepatocellular carcinoma: Chemoembolization improves survival. Hepatology 37: 429-442, 2003.

5. Lencioni R, Chen XP, Dagher L and Venook AP: Treatment of intermediate/advanced hepatocellular carcinoma in the clinic: How can outcomes be improved? Oncologist 15 (Suppl 4): 42-52, 2010.

6. Kawano Y, Sasaki A, Kai S, Endo Y, Iwaki K, Uchida H, Shibata K, Ohta M and Kitano S: Short- and long-term outcomes after hepatic resection for hepatocellular carcinoma with concomitant esophageal varices in patients with cirrhosis. Ann Surg Oncol 15: 1670-1676, 2008.

7. Saab S, Yeganeh M, Nguyen K, Durazo F, Han S, Yersiz H, Farmer DG, Goldstein LI, Tong MJ and Busuttil RW: Recurrence of hepatocellular carcinoma and hepatitis B reinfection in hepatitis B surface antigen-positive patients after liver transplantation. Liver Transpl 15: 1525-1534, 2009.

8. Kato Y, Tanaka N, Kobayashi K, Ikeda T, Hattori N and Nonomura A: Growth of hepatocellular carcinoma into the right atrium. Report of five cases. Ann Intern Med 99: 472-474, 1983.

9. Kojiro M, Nakahara H, Sugihara S, Murakami T, Nakashima T and Kawasaki $\mathrm{H}$ : Hepatocellular carcinoma with intra-atrial tumor growth. A clinicopathologic study of 18 autopsy cases. Arch Pathol Lab Med 108: 989-992, 1984.
10. Fujisaki M, Kurihara E, Kikuchi K, Nishikawa K and Uematsu Y: Hepatocellular carcinoma with tumor thrombus extending into the right atrium: Report of a successful resection with the use of cardiopulmonary bypass. Surgery 109: 214-219, 1991.

11. Zeng ZC, Fan J, Tang ZY, Zhou J, Qin LX, Wang JH, Sun HC, Wang BL, Zhang JY, Jiang GL and Wang YQ: A comparison of treatment combinations with and without radiotherapy for hepatocellular carcinoma with portal vein and/or inferior vena cava tumor thrombus. Int J Radiat Oncol Biol Phys 61: 432-443, 2005.

12. Ohwada S, Tanahashi Y, Kawashima Y, Satoh Y, Nakamura S, Kobayashi I, Ohya T, Ishikawa S, Ohtaki A, Iino Y, et al: Surgery for tumor thrombi in the right atrium and inferior vena cava of patients with recurrent hepatocellular carcinoma. Hepatogastroenterology 41: 154-157, 1994.

13. Kanematsu M, Imaeda T, Minowa H, Yamawaki Y, Mochizuki R, Goto H, Seki M, Doi H and Okumura S: Hepatocellular carcinoma with tumor thrombus in the inferior vena cava and right atrium. Abdom Imaging 19: 313-316, 1994.

14. Le Treut YP, Hardwigsen J, Ananian P, Saïsse J, Grégoire E, Richa $\mathrm{H}$ and Campan P: Resection of hepatocellular carcinoma with tumor thrombus in the major vasculature. A European case-control series. J Gastrointest Surg 10: 855-862, 2006.

15. Wang Y, Yuan L, Ge RL, Sun Y and Wei G: Survival benefit of surgical treatment for hepatocellular carcinoma with inferior vena cava/right atrium tumor thrombus: Results of a retrospective cohort study. Ann Surg Oncol 20: 914-922, 2013.

16. Chern MC, Chuang VP, Cheng T, Lin ZH and Lin YM: Transcatheter arterial chemoembolization for advanced hepatocellular carcinoma with inferior vena cava and right atrial tumors. Cardiovasc Intervent Radiol 31: 735-744, 2008.

17. Taura N, Hamasaki K, Nakao K, Ichikawa T, Nishimura D, Goto T, Fukuta M, Kawashimo H, Miyaaki H, Fujimoto M, et al: Aging of patients with hepatitis $C$ virus-associated hepatocellular carcinoma: Long-term trends in Japan. Oncol Rep 16: 837-843, 2006.

18. Fan HL, Chen TW, Hsieh CB, Jan HC, His SC, De-Chuan C, $\mathrm{Chu} \mathrm{CH}$ and $\mathrm{Yu}$ JC: Liver transplantation is an alternative treatment of hepatocellular carcinoma beyond the Milan criteria. Am J Surg 200: 252-257, 2010.

19. Nam SW, Baek JT, Kang SB, Lee DS, Kim JI, Cho SH, Park SH, Han JY, Ahn BM, Kim JK and Chung KW: A case of the hepatocellular carcinoma during the pregnancy and metastasis to the left atrium. Korean J Hepatol 11: 381-385, 2005.

20. Chong VH, Jamaludin AZ, Lim KS, Abdullah HM and Nair RT: Hepatocellular carcinoma with intravascular and right atrial extension. Indian J Gastroenterol 27: 255, 2008.

21. Tanaka A, Morimoto T, Ozaki N, Ikai I, Yamamoto Y, Tsunekawa S, Kitai T and Yamaoka Y: Extension of surgical indication for advanced hepatocellular carcinoma: Is it possible to prolong life span or improve quality of life? Hepatogastroenterology 43: 1172-1181, 1996.

22. Cheng HY, Wang XY, Zhao GL and Chen D: Imaging findings and transcatheter arterial chemoembolization of hepatic malignancy with right atrial embolus in 46 patients. World J Gastroenterol 14: 3563-3568, 2008.

23. Inoue $Y$, Hayashi M, Katsumata T, Shibayama $Y$ and Tanigawa $N$ : Hepatocellular carcinoma with right atrial tumor thrombus: Report of a case. Surg Today 41: 1122-1129, 2011.

24. Lin SM, Lin CJ, Lin CC, Hsu CW and Chen YC: Randomised controlled trial comparing percutaneous radiofrequency thermal ablation, percutaneous ethanol injection, and percutaneous acetic acid injection to treat hepatocellular carcinoma of $3 \mathrm{~cm}$ or less. Gut 54: 1151-1156, 2005.

25. Itoh S, Ikeda Y, Kawanaka H, Okuyama T, Kawasaki K, Eguchi D, Korenaga D and Takenaka K: Efficacy of surgical microwave therapy in patients with unresectable hepatocellular carcinoma. Ann Surg Oncol 18: 3650-3656, 2011.

26. Takayasu K, Arii S, Ikai I, et al: Prospective cohort study of transarterial chemoembolization for unresectable hepatocellular carcinoma in 8510 patients. Gastroenterology 131: 461-469, 2006.

27. Xue TC, Xie XY,Zhang L, Yin X, Zhang BH and Ren ZG: Transarterial chemoembolization for hepatocellular carcinoma with portal vein tumor thrombus: A meta-analysis. BMC Gastroenterol 13: 60, 2013.

28. Nishikawa H, Osaki Y, Kita R and Kimura T: Hepatic arterial infusion chemotherapy for advanced hepatocellular carcinoma in Japan. Cancers (Basel) 4: 165-183, 2012.

29. Miyazaki M, Suzuki H, Itoh H, et al: Portal vein infusion of cancer chemotherapeutic agent emulsified with Lipiodol in regenerating liver after partial hepatectomy in rats. Res Exp Med (Berl) 193: 231-240, 1993. 